\title{
Lesão Renal Aguda por Glicerol: Efeito Antioxidante da Vitis Vinifera $L^{*}$
}

\author{
Acute Kidney Injury by Glycerol: \\ Antioxidant Effect of Vitis Vinifera $L$.
}

Elisabete Cristina de Oliveira Martim¹ ${ }^{1}$,Carolina Ferreira Pinto², Mirian Watanabe ${ }^{3}$, Maria de Fátima Fernandes Vattimo ${ }^{4}$

\section{RESUMO}

JUSTIFICATIVA E OBJETIVOS: A lesão renal aguda (LRA) é a complicação mais grave da rabdomiólise. Nessa síndrome, a liberação do pigmento heme desencadeia uma lesão que se caracteriza por vasoconstrição glomerular e toxicidade celular direta com provável componente oxidante. A renoproteção com antioxidantes tem demonstrado efeito satisfatório. As proantocianidinas são antioxidantes naturais encontradas no extrato da semente da uva. O objetivo deste estudo foi avaliar o efeito antioxidante da Vitis vinifera sobre a função renal de ratos submetidos à lesão induzida por rabdomiólise.

MÉTODO: Foram utilizados ratos Wistar, machos e adultos pesando entre 250 e $300 \mathrm{~g}$. A LRA foi induzida pela administração de glicerol $50 \%$ por via muscular. Os animais foram distribuídos em 4 grupos: grupo Salina $(6 \mathrm{~mL} / \mathrm{kg}$ de $\mathrm{NaCl}$ a $0,9 \%)$, por via intraperitoneal (dose única), Glicerol (6 mL/kg) por via muscular metade da dose em cada região femoral, em dose única,

1. Enfermeira Mestre em Enfermagem

2. Mestre pela Escola de Enfermagem da USP.

3. Mestre e Doutoranda da Escola de Enfermagem da USP.

2. Professora Associada do Departamento de Enfermagem Médico Cirúrgica da Escola de Enfermagem da USP

*Recebido do Departamento de Enfermagem Médico-Cirúrgica da Escola de Enfermagem da Universidade de São Paulo (USP), São Paulo, SP

Apresentado em 30 de julho de 2007

Aceito para publicação em 10 de setembro de 2007

Endereço para correspondência:

Elisabete Cristina de Oliveira Martim

Av. Dr. Enéas de Carvalho Aguiar, 419.

05403-000 São Paulo, SP

Fone: (11) 3061-7549

E-mail: nephron@usp.br

E-mail: elisabetecris@terra.com.br

(C)Associação de Medicina Intensiva Brasileira, 2007 grupo Vitis vinifera (3 mg/kg/dia), por via oral durante cinco dias e grupo Glicerol + Vitis vinifera que recebeu Vitis vinifera por cinco dias antes do glicerol.

RESULTADOS: Foram avaliados a função renal (FR) e o perfil oxidativo (peróxidos urinários FOX-2 e MDATBARS). O grupo glicerol de animais tratado com Vitis vinifera apresentou melhora da FR e redução dos níveis de peroxidação lipídica.

CONCLUSÕES: Os resultados deste estudo confirmaram a ação antioxidante da Vitis vinifera na LRA induzida por glicerol.

Unitermos: Antioxidante, Favonóides Lesão Renal Aguda, Proantocianidinas, Rabdomiólise

\section{SUMMARY}

BACKGROUND AND OBJECTIVES: The Acute Kidney Injury (AKI) is the most serious complication of rhabdomyolysis. In this syndrome, the delivery of heme pigment induces an injury that distinguishes itself by glomerular vasoconstriction and direct cellular toxicity with oxidative component. The renoprotection with antioxidants has demonstrated satisfactory effect. The proanthocyanidins are natural antioxidants found in the grape seed extract. The aim of this study was to evaluate the antioxidant effect of Vitis vinifera (grape seed extract) on the renal function of rats submitted to the injury by rhabdomyolysis.

METHODS: Wistar rats, male, adults, weight ranging from 250-300g were used. The AKI was induced by intramuscular administration of glycerol $50 \%$. The animals were distributed in 4 groups: Saline group $(6 \mathrm{~mL} /$ $\mathrm{kg}$ of $\mathrm{NaCl} 0.9 \%$ intraperitoneal once a day), Glycerol group ( $6 \mathrm{~mL} / \mathrm{kg}$ ) of intramuscular glycerol each femoral region received $3 \mathrm{~mL} / \mathrm{kg}$ of glycerol, once a day), Vitis vinifera group ( $3 \mathrm{mg} / \mathrm{kg} /$ day v.o by 5 days) and Glycerol + Vitis vinifera by 5 days before glycerol).

RESULTS: Renal function (RF-creatinine clearance) and oxidative profile (urinary peroxides-FOX-2 and 
MDA-TBARS) were evaluted. The Glycerol group treated with Vitis vinifera has shown improvements in RF and reduction levels of lipid peroxidation.

CONCLUSION: The results of this study have confirmed the antioxidant protection of Vitis vinifera in AKI induced by glycerol.

Key Words: Acute Kidney Injury, Antioxidant, Flavonoids, Proanthocyanidins, Rhabdomyolysis

\section{INTRODUÇÃO}

A lesão renal aguda (LRA) é a complicação mais importante no curso da rabdomiólise. A rabdomiólise decorre primeiramente de necrose muscular caracterizando a causa traumática.

Entre os mecanismos propostos para explicar a associação entre a rabdomiólise e a LRA destacam-se: obstrução tubular pela presença de cilindros formados pelo pigmento heme, a vasoconstrição renal e a toxicidade direta da proteína heme sobre a célula tubular e mecanismos inter-relacionados ${ }^{1,2}$.

A necrose muscular desencadeia o distúrbio oxidativo pela alteração na homeostase do cálcio, depleção de ATP e ativação de sistemas enzimáticos como proteases e fosfolipases que irão participar da formação e liberação de radicais livres, resultando em lesão celular².

A lesão oxidativa altera a estrutura e função glomerular por efeito das espécies reativas de oxigênio (EROs) em células mesangias e endoteliais, desencadeando lesão inflamatória causada pela liberação de mediadores como citocinas e a ativação leucocitária, além da redução de oxido nítrico (NO) implicando no relaxamento vascular, por fim a ativação da peroxidação lipídica ${ }^{3,4}$.

Os antioxidantes naturais podem auxiliar na resposta endógena além de contribuir para depuração das EROs, atenuando seu efeito oxidativo no rim $^{5,6}$. A Organização Mundial da Saúde (OMS) já reconhece, na atualidade, a importância da fitoterapia, sugerindo ser uma alternativa viável e importante particularmente para os países em desenvolvimento, entre outras características, pelo baixo custo. Pesquisas realizadas em universidades brasileiras já identificaram mais de 350 mil espécies vegetais, com usos medicinais diversos.

A Vitis vinifera $L$. é um antioxidante natural extraído das sementes de uva, seus componentes ativos constituem flavonóides e proantocianidinas, que além de atuarem como seqüestradores de radical livre, promo- vem a vasodilatação, inibem enzimas como fosfolipase, ciclooxigenase e lipooxogenase, além de reduzir a peroxidação lipídica ${ }^{7,8}$.

O objetivo deste estudo foi verificar o efeito do précondicionamento com Vitis vinifera sobre a função renal e o perfil oxidativo em ratos submetidos à rabdomiólise com a administração por via muscular de glicerol a $50 \%$.

\section{MÉTODO}

Foram utilizados ratos da raça Wistar, adultos, machos pesando entre 250 e $300 \mathrm{~g}$, divididos em 4 grupos:

- Grupo Salina: animais que receberam $6 \mathrm{~mL} / \mathrm{kg}$ de cloreto de sódio $(\mathrm{NaCl}$ a $0,9 \%)$, por via intraperitoneal em dose única;

- Grupo glicerol: animais que receberam glicerol a $50 \%$ (6 mL/kg - Vitaly - farmácia de manipulação e homeopatia), por via muscular, metade da dose em cada região femoral, em dose única;

- Grupo Vitis vinifera: animais que receberam Vitis vinifera (3 mg/kg/dia - Vittis ${ }^{\circledR}$ - Herbarium Laboratório Botânico Ltda), por via oral durante cinco dias;

- Grupo glicerol + Vitis vinifera que receberam durante cinco dias pré-condicionamento com Vitis vinifera (3 $\mathrm{mg} / \mathrm{kg} / \mathrm{dia}$ ) por via oral antes da administração de glicerol a $50 \%(6 \mathrm{~mL} / \mathrm{kg})$, por via muscular.

Os animais "Vitis" receberam o próprio extrato do fitoterápico que é comercialmente disponível. Os componentes ativos são as proantocianidinas oligoméricas também conhecidas como as proantocianidinas ou oligômeros "procianidólicos", que são os principais responsáveis pelos efeitos farmacológicos. Esses compostos pertencem à família dos bioflavonóides. As proantocianidinas mais potentes são aquelas ligadas a outras proantocianidinas, como por exemplo uma mistura de proantocianidinas como aquela utilizada neste estudo. A dose diária recomendada em humanos é 150-300 mg, onde cada cápsula contém 135 mg de proantocianidinas, cujo extrato está padronizado em 95\% de proantocianidinas.

Os animais foram colocados em gaiola metabólica por 24 horas para coleta de urina. Foram anestesiados no dia seguinte para coleta de sangue através da punção da aorta abdominal.

Ao final do experimento, os animais foram sacrificados de acordo com as recomendações para a utilização de animais em estudos científicos, em que se instituiu a inalação de éter dietílico até a parada respiratória total. 


\section{- Avaliação renal}

A avaliação da função renal foi realizada por meio da mensuração da taxa de depuração de creatinina (CLcr). Utilizou-se o método de Jaffé para a mensuração de creatina plasmática e urinária e a leitura foi realizada em espectrofotometria (Beckman) em absorbância de $521 \mathrm{~nm}$.

\section{- Método FOX-2 para peróxidos urinários}

O método de FOX consiste na determinação dos níveis de peróxido por meio do método ferro-xylenol orange (XO). Os peróxidos orgânicos são um dos primeiros produtos semi-estáveis da interação entre radicais livres e outras espécies reativas de oxigênio no sistema biológico. Os peróxidos são considerados como potenciais indicadores na formação e ou resultantes das moléculas reativas.

Para a mensuração dos peróxidos urinários foi utilizado o método FOX. A leitura das amostras urinárias foi realizada em espectrofotometria (Beckman) em absorbância de $560 \mathrm{~nm}$, e os valores foram calculados pelo coeficiente para peróxidos de hidrogênio de 4,3 x $10 \mathrm{Mcm}$ e estabilizados para grama de creatinina urinária.

\section{- Dosagem de TBARS (substâncias reativas com ácido tiobarbitúrico) na urina}

O MDA é um dos aldeídos freqüentemente analisados em métodos analíticos quantitativos e qualitativos para determinação dos índices de peroxidação lipídica. O MDA é um dos principais produtos da cascata de peroxidação lipídica e pode ser detectado por vários métodos dentre eles por meio da luz ultravioleta em sistema de cromoterapia de alta pressão (HPLC) e também com o do uso do ácido tiobarbitúrico ao qual reage com várias substâncias dentre elas o MDA (TBARS). A leitura foi realizada em espectrofotometria (Beckman) em absorbância de 560 nm, e os valores de MDA apresentados foram calculados por meio do coeficiente de extinção molar $1,56 \times 10^{5} \mathrm{~cm}^{-1}$.

\section{- Análise estatística}

Utilizou-se o método GLM (ANOVA univariada) e os testes múltiplos de comparação Bonferroni ajustado. Os resultados foram apresentados como média \pm erro padrão (SEM). Os valores de $\mathrm{p}<0,005$ foram considerados significativos.

\section{RESULTADOS}

A tabela 1 ilustra a diferença significativa quanto ao fluxo urinário do grupo Glicerol quando comparado ao grupo Salina. A elevação no fluxo também ocorreu no grupo Glicerol + Vitis vinifera, porém quando esse foi comparado com seu controle não se constatou diferença significativa. Esse achado pode estar relacionado ao aumento voluntário da ingesta hídrica dos animais do grupo Glicerol (Glicerol $56 \pm 20,05$ versus Salina 36 $\pm 11 \mathrm{~mL} / 24 \mathrm{~h}$ ) de água por animal.

A administração de glicerol a $50 \%$ por via muscular reproduziu o modelo de LRA por rabdomiólise, resultando na redução significativa do Clcr (depuração de creatinina) quando comparado ao grupo Salina (Glicerol 0,20 $\pm 0,02$ versus Salina 0,65 $\pm 0,03$ ).

O tratamento prévio com Vitis vinifera determinou proteção funcional renal significativa nos animais que receberam glicerol $(0,51 \pm 0,02$ versus $0,20 \pm 0,02)$, tendo restituído valores de Clcr similares ao grupo Vitis vinifera e muito próximos aos do grupo Salina.

Quanto aos peróxidos urinários, o grupo Glicerol quando comparado ao grupo Salina apresentou elevação estatisticamente significativa na excreção de peróxidos urinários $(20 \pm 0,8$ versus $4,2 \pm 0,4)$.

O grupo Glicerol + Vitis vinifera quando comparado ao grupo Glicerol apresentou redução significativa dos peróxidos urinários $(14,9 \pm 1$ versus $20 \pm 0,8)$, porém, apesar de visível melhora, os resultados não se igualaram àqueles expressos para o grupo Vitis vinifera $(14,9$ \pm 1 versus $7,1 \pm 0,4$ )

Tabela 1 - Função Renal Global, Peróxidos Urinários e TBARs dos Grupos Estudados

\begin{tabular}{|c|c|c|c|c|c|}
\hline Grupos & $\mathrm{n}$ & $\begin{array}{l}\text { Fluxo Urinário } \\
(\mathrm{mL} / \mathrm{min})\end{array}$ & $\begin{array}{l}\text { Depuração / } 100 \mathrm{~g} \\
(\mathrm{~mL} / \mathrm{min})\end{array}$ & $\begin{array}{l}\text { Peróxidos Urinários } \\
\text { (nmol/g de creatinina) }\end{array}$ & $\begin{array}{l}\text { TBARS } \\
\text { (nmol de TBARS urinários/24 h) }\end{array}$ \\
\hline Salina & 6 & $0,007 \pm 0,003$ & $0,65 \pm 0,03$ & $4,2 \pm 0,4$ & $149,6 \pm 24,0$ \\
\hline Glicerol & 8 & $0,020 \pm 0,002$ & $0,20 \pm 0,02^{\wedge}$ & $20,0 \pm 0,8^{\mathbf{\Delta}}$ & $380,9 \pm 22,4 \mathbf{\Delta}$ \\
\hline Vitis vinifera & 5 & $0,007 \pm 0,003$ & $0,57 \pm 0,03$ & $7,1 \pm 0,4$ & $126,4 \pm 28,3$ \\
\hline Glicerol+ Vitis vinifera & 8 & $0,015 \pm 0,002$ & $0,51 \pm 0,02^{\square \diamond}$ & $14,9 \pm 1,0^{\Delta \Delta o}$ & $233,0 \pm 24,0^{\diamond}$ \\
\hline
\end{tabular}

Os dados representam médias \pm erro padrão ( SEM) $\wedge^{\wedge} p<0,001$ vs Salina, ${ }^{\natural} p<0,005$ vs Salina, ${ }^{\natural} p<0,001$ vs Glicerol, ${ }^{\natural} p<0,003$ vs Glicerol, $" p<0,01$ vs Vitis vinifera, ${ }^{\Delta} \mathrm{p}<0,001$ vs Vitis vinifera, ${ }^{\circ} \mathrm{p}<0,004$ vs Glicerol. 
Com relação ao MDA urinário o grupo Glicerol + Vitis vinifera apresentou redução dos níveis de MDA quando comparado ao grupo Glicerol $(233 \pm 24$ versus $380 \pm$ $22,4)$. Foi identificado também que não houve estatística significativa entre esses e os demais grupos controle, sublinhando a ação antioxidante do fitoterápico testado.

\section{DISCUSSÃo}

A fisiopatologia da mioglobinúria induzida pela administração por via muscular de glicerol a $50 \%$ constituiu-se em modelo clássico de LRA experimental. A lesão muscular causada com a administração do glicerol levou a miólise que está relacionada à deterioração da função e estrutura renal. Os mecanismos fisiopatológicos envolvidos são a obstrução tubular, a vasoconstrição renal e o estresse oxidativo².

Dentre as várias ações deletérias da mioglobina envolvidas no processo da LRA por rabdomiólise, sabe-se que a liberação de ferro dos estoques celulares estimula a conversão de $\mathrm{H}_{2} \mathrm{O}_{2}$ em $\mathrm{OH}$, gerando maior quantidade desse radical hidroxila e conseqüentemente intensificando a peroxidação lipídica ${ }^{2,9}$.

Os resultados deste estudo mostraram que a administração de glicerol a 50\% em ratos desencadeou LRA caracterizada pela diminuição da função renal representada pela diminuição dos níveis da depuração de creatinina.

A disfunção tubular determinada pela heme está pautada na toxicidade direta daquele pigmento sobre a célula epitelial. Durante a mioglobinúria a lesão tubular proximal é decorrente de dois fatores que apresentam papel critico: lesão isquêmica mediada pela habilidade do pigmento heme em intensificar a vasoconstrição renal e o estresse oxidativo mediado pelas EROs ${ }^{10-12}$.

Estudos têm referendado o uso de fitoterápicos no tratamento e prevenção de doenças crônicas e estresse oxidativo. Neste contexto, o uso de agentes oxidantes naturais oriundos da dieta e bebidas como o vinho tem merecido destaque por possuir propriedades relacionadas ao seqüestro radicais livres, quelação de metais e modulação de atividades enzimáticas em prol da inibição da peroxidação lipídica, presentes em vários processos patológicos, como a doença renal ${ }^{13-15}$.

Neste cenário, os flavonóides do vinho representam o grupo de plantas fenólicas que exercem ação antioxidante relevante. De todos os componentes presentes na Vitis vinifera, os compostos fenólicos, especialmen- te as proantocianidinas, têm despertado interesse em estudos clínicos e experimentais. As proantocianidinas são antioxidantes naturais encontradas no extrato da semente da uva ${ }^{5,6}$.

Estudos recentes demonstraram que o uso de um bioflavonóide proantocianidina em modelo de lesão renal por glicerol, também extraído das sementes de uvas, melhorou significativamente a histologia renal com redução do volume tubular e dos restos celulares tubulares. Foi identificada também recuperação da função renal e sugerido que a melhora histo-funcional poderia ser atribuída ao relaxamento vascular determinado pela protoantocianidina. Contudo, no estudo citado não foram realizados de mensuração de peroxidação lipídica ${ }^{16}$.

Em modelo de isquemia também pôde ser observado o efeito renoprotetor das protoantocianidas. Demonstrou-se que o pré-tratamento com uma mistura de bioflavonóides em ratos submetidos à isquemia renal bilateral protegeu a função com elevação da depuração de creatinina e redução da creatinina plasmática. Observou-se ainda melhora histológica caracterizada por redução da necrose tubular na faixa externa da medula externa ${ }^{17}$.

No presente estudo o efeito renoprotetor da Vitis vinifera se confirmou. O pré-tratamento com o fitoterápico mostrou melhora significativa da depuração de creatinina. Os dados referentes à peroxidação lipídica ressaltaram o efeito antioxidante da Vitis vinifera.

Neste estudo, o ensaio sobre o papel antioxidante da Vitis vinifera mostrou, com relação à dosagem de peróxidos e MDA urinários, a redução dos níveis da peroxidação lipídica, fortalecendo a hipótese relativa ao efeito antioxidante da Vitis vinifera.

Em síntese, a Vitis vinifera confirmou de forma inquestionável o efeito renoprotetor provavelmente relacionado ao seu efeito antioxidante. Ressalte-se o uso em pacientes de risco para lesão renal aguda, como aqueles de terapia intensiva, cujos agravos e exposição às toxinas são diversos e nos quais a prevalência dessa síndrome é particularmente constrangedora (até 70\%). Estudos que avancem nessa investigação utilizando outros modelos de lesão em diferentes sujeitos, serão de grande valor para melhor compreensão dos ramos da lesão e alternativas terapêuticas na rabdomiólise.

Os resultados deste estudo demonstraram melhora da função renal associada à redução dos níveis de peroxidação lipídica, fortalecendo a hipótese relativa ao efeito antioxidante da Vitis vinifera. 


\section{REFERÊNCIAS}

01. Paller MS - Hemoglobin- and myoglobin-induced acute renal failure in rats: role of iron in nephrotoxicity. Am J Physiol, 1988;255:F539-F544.

02. Zager RA - Rhabdomyolysis and myohemoglobinuric acute renal failure. Kidney Int, 1996;49:314-326.

03. Rovin BH, Phan LT - Chemotactic factors and renal inflammation. Am J Kidney Dis, 1998;31:1065-1084.

04. Tak PP, Firestein GS - NF-kappaB: a key role in inflammatory diseases. J Clin Invest, 2001;107:7-11.

05. Rodrigo R, Bosco C, Herrera P et al - Amelioration of myoglobinuric renal damage in rats by chronic exposure to flavonol-rich red wine. Nephrol Dial Transplant, 2004;19:2237-2244.

06. Stefanovic V, Savic V, Vlahovic P et al - Reversal of experimental myoglobinuric acute renal failure with bioflavonoids from seeds grape. Ren Fail, 2000;22:255-266.

07. Salah N, Miller NJ, Paganga G et al - Polyphenolic flavanal as scavengers of aqueous phase radicals and chain-breaking antioxidants. Arch Biochem Biophys, 1995;322:339-346.

08. Rice-Evans CA, Miller NJ, Paganga G - Structure-antioxidant activity relationship of flavonoids and phenolic acids. Free Radic Biol Med, 1996;20:933-956.

09. Knochel $\mathrm{J}$ - Rhabdomyolysis and myoglobinuria. Ann Rev Med, 1992;33:435-443.
10. Brezis M, Heyman SN, Dinour D et al - Role of nitric oxide in renal medullary oxygenation. Studies in isolated and intact rat kidneys. J Clin Invest, 1991;88:390-395.

11. Nath KA, Balla G, Vercellotti MG et al - Induction of heme oxygenase is a rapid, protective response in rhabdomyolysis in rat. J Clin Invest, 1992;90:267-270.

12. Sharma VS, Traylor TG, Gardiner R - Reaction of nitric oxide with heme proteins and model compounds of hemoglobin. Biochemistry, 1987;26:3837-3843.

13. Morillas-Ruiz JM, Villegas Garcia JA, Lopez FJ et al - Effects of polyphenolic antioxidants on exercise-induced oxidative stress. Clin Nutr, 2006;25:444-453.

14. Puiggros F, Llopiz N, Ardevol A et al - Grape seed procyanidins prevent oxidative injury by modulating the expression of antioxidant enzyme systems. J Agric Food Chem, 2005;53:6080-6086.

15. Simonetti P, Ciappellano S, Gardana C et al - Procyanidins from Vitis vinifera seeds: in vivo effects on oxidative stress. J Agric Food Chem, 2002;50:6217-6221.

16. Avramovic V, Vlahovic P, Mihailovic D et al - Protective effect of a bioflavonoidd proanthocyanidin-BP1 in glycerol-induced acute renal failure in the rats: renal stereological study. Ren Fail, 1999;21:627-634.

17. Ohkita M, Nakajima A, Ueda K et al - Preventive effect of flavangenol on ischemia/reperfusion-induced acute renal failure in rats. Biol Pharm Bull, 2005;28:1655-1657. 